\title{
Self-help manuals may reduce bingeing and purging in bulimia nervosa
}

Carter JC, Olmsted MP, Kaplan AS et al. Self-help for bulimia nervosa: a randomized controlled trial. Am J Psychiatry 2003;160:973-8.

\section{QUESTION: Do self help manuals improve symptoms in people with bulimia nervosa?}

\section{Design}

Randomised controlled trial.

\section{Setting}

Specialist hospital clinic, Toronto, Canada.

\section{Participants}

85 women with bulimia nervosa $(93 \%$ with the purging subtype), mean age 27 years (range $17-53$ years), waiting for treatment at a hospital clinic. Exclusion criteria were: previous specialist treatment for bulimia nervosa; bingeing or purging symptoms less than once a week; body mass index less than 18; other medical conditions, or pregnancy.

\section{Intervention}

8 weeks of treatment with: self-help manual on cognitive-behavioural skills specifically designed for bulimia nervosa (cognitive-behaviour manual); nonspecific self-help manual on self-assertiveness skills (unguided self help manual), or waiting list control.

\section{Main outcome measures}

Response was defined as a $50 \%$ or greater reduction in frequency of binge eating or purging as assessed by the Eating Disorders Examination interview.

\section{Main results}

20 participants did not complete the study. Intention-totreat analysis found that either self-help intervention increased response rate compared with control. However, results did not reach statistical significance, perhaps due to a lack of study power (response rate: $54 \%$ with cognitive behaviour manual; $50 \%$ with unguided self-help manual; $31 \%$ with control; $p=0.10$ for cognitive behaviour manual $v$ control; $\mathrm{p}=0.08$ for unguided self-help manual $v$ control).

\section{Conclusions}

Self-help manuals may benefit people with bulimia nervosa.

\section{COMMENTARY}

Alternative approaches to healing bulimia nervosa should be promoted. Self-help is one such approach, which provides advantages in terms of economics, accessibility, delivery and empowerment of the patient. ${ }^{1-2}$ With these assumptions, Carter et al conducted a pioneering, randomised, controlled trial study of the effectiveness of a self-help approach at the initial stage of treatment for clients suffering from bulimia. The implications of this study for clinical practice are three-fold.

First, shifting from an expert-led to client-led ideology and modality for treating bulimia highlights the importance of empowerment and participation of patients in the management of bulimic symptoms. This is the core insight gained from the research. Second, user-friendly self-help manuals for patients can be substituted in place of more expensive psychiatric or other professional services. Finally, benefits of teaching both cognitive behaviour self-help skills and self-assertion skills are similar. This suggests the potential of non-specific self-help skills to minimise the symptoms of bulimia while the patient is waiting for psychiatric treatment. This study also provides guidelines and ready-made resources for primary treatment personnel - including general practitioners, social workers, nurses and teachers - when they are faced with bulimic patients awaiting formal mental health services.

Zenobia C Y Chan, RN MPHC PhD Candidate Department of Social Work The Chinese University of Hong Kong

1 Baker CW, Brownell, KD. Binge eating disorder: Identification and management. Nutr Clin Care 1999; 2: 344-353.

2 Wilson GT, Loeb KL. Stepped care treatment for eating disorders. J Consul Clin Psychol 2000; 68: 564-572.
Sources of funding: The Dean's Fund, Faculty of Medicine, University of Toronto.

Correspondence to: Dr Carter, Department of Psychiatry, Toronto General Hospital, 200 Elizabeth St,Eaton

Wing, 8-231, Toronto, Ont.M5G 2C4

Canada. email: jacqueline.carter@ uhnon.ca 